Tôhoku Math. Journ.

27 (1975), 31-47.

\title{
GENERATORS AND MAXIMAL IDEALS IN SOME ALGEBRAS OF HOLOMORPHIC FUNCTIONS
}

\author{
Niro YaNAGIHARA
}

(Received October 2, 1973)

1. Introduction. Let $D$ be the unit disk $\{|z|<1\}$. A holomorphic function $f(z)$ in $D$ is said to belong to the class $N$ of functions of bounded characteristic if

$$
T(r, f)=\frac{1}{2 \pi} \int_{0}^{2 \pi} \log ^{+}\left|f\left(r e^{i \theta}\right)\right| d \theta=O(1) \text { as } r \rightarrow 1
$$

A function $f(z) \in N$ is said to belong to the class $N^{+}[2$, p. 25] if

$$
\lim _{r \rightarrow 1} \int_{0}^{2 \pi} \log ^{+}\left|f\left(r e^{i \theta}\right)\right| d \theta=\int_{0}^{2 \pi} \log ^{+}\left|f\left(e^{i \theta}\right)\right| d \theta .
$$

The class $N^{+}$can be considered as an $F$-space in the sense of Banach $[1$, p. 51], with the metric [9]

$$
\rho(f, g)=\frac{1}{2 \pi} \int_{0}^{2 \pi} \log \left(1+\left|f\left(e^{i \theta}\right)-g\left(e^{i \theta}\right)\right|\right) d \theta \text { for } f, g \in N^{+} .
$$

$N^{+}$is easily seen to be a topological algebra with respect to this metric (1.3). $\quad N^{+}$is neither locally convex nor locally bounded, but has sufficiently many continuous linear functionals to form a dual system $\left\langle\left(N^{+}\right)^{*}, N^{+}\right\rangle$in the sense of Dieudonné and Mackey [7, p. 88].

On the other hand, we defined a Fréchet space $F^{+}$which contains $N^{+}$ [9]. We say that a holomorphic function $f(z)$ in $D$ belongs to the class $F^{+}$if

$$
M(r, f)=\max _{|z|=r}|f(z)| \leqq K_{f} \exp \left[\omega_{f}(r) /(1-r)\right]
$$

with a constant $K_{f}>0$ and a continuous function $\omega_{f}(r), 0 \leqq r<1$, depending on $f \in F^{+}$, such that $\omega_{f}(r) \downarrow 0$ as $r \rightarrow 1$. A holomorphic function $f(z)=\sum a_{n} z^{n}$ belongs to $F^{+}$if and only if

$$
\|f\|_{F c}=\int_{0}^{1} \exp \left[\frac{-c}{1-r}\right] M(r, f) d r<\infty
$$

for each $c>0$. (1.4) is equivalent to

$$
a_{n}=O(\exp [o(\sqrt{n})]) \quad \text { as } n \rightarrow \infty \text {. }
$$


$F^{+}$is a countably normed (locally convex) Fréchet space with the system of (semi-)norms $\left\{\|\cdot\|_{F c}\right\}_{c>0} \cdot F^{+}$is the second dual space for the space $N^{+}$ [11], and is a nuclear as well as a Montel space [11]. We can easily see that $F^{+}$is a topological algebra.

In this note, we will characterize generators of the algebra $\mathrm{F}^{+}$, following to the methods of Hörmander [3], Kelleher and Taylor [5], [6]. Although they treat mainly with several variables, we confine here ourselves only to one variable case. Generalizations to several variables are concerns of our further study.

In $\S \S 5-6$, we will determine closed and other maximal ideals in $F^{+}$.

2. Generators for $\boldsymbol{F}^{+}$. Let $f_{1}, \cdots, f_{N} \in F^{+}$. The ideal in $F^{+}$generated by $\vec{f}=\left(f_{1}, \cdots, f_{N}\right)$ is denoted as $I\left(f_{1}, \cdots, f_{N}\right)$. We write

$$
\|\vec{f}(z)\|^{2}=\left|f_{1}(z)\right|^{2}+\cdots+\left|f_{N}(z)\right|^{2}, \quad z \in D .
$$

If $u \in F^{+}$belongs to the ideal $I\left(f_{1}, \cdots, f_{N}\right)$, then it is easily seen that there exist a constant $K>0$ and a continuous function $\omega(r), \omega(r) \downarrow 0$ as $r \rightarrow 1$, such that

$$
|u(z)| \leqq K\|\vec{f}(z)\| \exp [\omega(r) /(1-r)], \quad|z|=r .
$$

THEOREM 1. If $u \in F^{+}$satisfies (2.2), then we have

$$
u^{2} \in I\left(f_{1}, \cdots, f_{N}\right) .
$$

As a corollary of Theorem 1 , we have

Theorem 2. Let $f_{1}, \cdots, f_{N} \in F^{+}$. In order that there exist $g_{1}, \cdots$, $g_{N} \in F^{+}$such that

$$
f_{1} g_{1}+\cdots+f_{N} g_{N}=1,
$$

it is necessary and sufficient that

$$
\left|f_{1}(z)\right|+\cdots+\left|f_{N}(z)\right| \geqq \delta \exp [-\omega(r) /(1-r)]
$$

$(r=|z|)$ for some constant $\delta>0$ and for some continuous function $\omega(r)$, $\omega(r) \downarrow 0$ as $r \rightarrow 1$.

In contrast to Theorem 1 , we have

Theorem 1*. (2.2) does not imply that $u \in I\left(f_{1}, \cdots, f_{N}\right)$ for $u \in F^{+}$.

For the proof, we follow to the method of Rao [8].

In connection with Theorem 2, we have

TheOREM $2^{*}$. Let $f_{1}, \cdots, f_{N} \in N^{+}$. Then, (2.4) is not sufficient for $f_{1}, \cdots, f_{N}$ to be generators of $N^{+}$. That is, (2.4) does not imply (2.3) in $N^{+}$. 
In contrast to the case of Banach algebras, we have

THEOREM 3. Maximal ideals in $F^{+}$are not necessarily closed.

Hence, in $\$ 6$, we will use somewhat strange method for compactifying in order to put it in a one-to-one correspondence with the maximal ideal space of $\mathrm{F}^{+}$.

\section{Proof of Theorem 1.}

LEMMA 1. Let $\omega_{1}(r)$ be a continuous function, $\omega_{1}(r) \downarrow 0$ as $r \rightarrow 1$. Then we can find a continuous function $\omega(r)$ such that $\omega(r) \geqq \omega_{1}(r)$ and $\omega(r) \geqq \sqrt{1-r}$,

$$
\omega(r) \downarrow 0, \quad \omega(r) /(1-r) \uparrow \infty \quad \text { as } r \rightarrow 1
$$

$\omega(r) /(1-r)$ is convex.

Proof. We can suppose that $\omega_{1}(r)$ is continuously differentiable and $\omega_{1}^{\prime}(r)<0$ for $0 \leqq r<1$.

Let $r_{0}=0$. Let $r_{1}$ be a number, $1 / 2<r_{1}<1$, and put

$$
\begin{aligned}
& a_{1}=\omega_{1}\left(r_{0}\right) /\left(1-r_{1}\right)^{2}, \\
& b_{1}=-a_{1} r_{1}+\omega_{1}\left(r_{0}\right) /\left(1-r_{1}\right) .
\end{aligned}
$$

Let $r_{2}$ be such that $r_{2}>r_{1}$ and

$$
a_{1} r_{2}+b_{1}=\omega_{1}\left(r_{1}\right) /\left(1-r_{2}\right) \text {. }
$$

Then $r_{2}<1$. Suppose $\left\{r_{k}\right\}_{k=0}^{n}, r_{k}<r_{k+1}$, and $\left\{a_{k}\right\}_{k=1}^{n-1},\left\{b_{k}\right\}_{k=1}^{n-1}$ be determined. Then, put

$$
\begin{aligned}
& a_{n}=\omega_{1}\left(r_{n-1}\right) /\left(1-r_{n}\right)^{2}, \\
& b_{n}=-a_{n} r_{n}+\omega_{1}\left(r_{n-1}\right) /\left(1-r_{n}\right),
\end{aligned}
$$

and let $r_{n+1}$ be such that $r_{n+1}>r_{n}$ and

$$
a_{n} r_{n+1}+b_{n}=\omega_{1}\left(r_{n}\right) /\left(1-r_{n+1}\right) \text {, }
$$

then $r_{n+1}<1$. We will show that $r_{n} \uparrow 1$. For that purpose, we put

$$
\rho=\lim _{n \rightarrow \infty} r_{n} \text {. }
$$

We have

$$
\begin{aligned}
a_{n} & =\left(r_{n+1}-r_{n}\right)^{-1}\left(\omega_{1}\left(r_{n}\right) /\left(1-r_{n+1}\right)-\omega_{1}\left(r_{n-1}\right) /\left(1-r_{n}\right)\right) \\
& =\frac{1}{1-r_{n+1}} \frac{\omega_{1}\left(r_{n}\right)-\omega_{1}\left(r_{n-1}\right)}{r_{n}-r_{n-1}} \frac{r_{n}-r_{n-1}}{r_{n+1}-r_{n}}+\frac{\omega_{1}\left(r_{n-1}\right)}{\left(1-r_{n+1}\right)\left(1-r_{n}\right)} .
\end{aligned}
$$

If $\rho$ in (3.3) would be $\rho<1$, we would have, letting $n \rightarrow \infty$ in (3.4), 


$$
\omega_{1}(\rho) /(1-\rho)^{2}=(1-\rho)^{-1} \omega_{1}^{\prime}(\rho) \lim _{n \rightarrow \infty} \frac{r_{n}-r_{n-1}}{r_{n+1}-r_{n}}+\omega_{1}(\rho) /(1-\rho)^{2}
$$

hence

$$
\lim _{n \rightarrow \infty}\left(\left(r_{n}-r_{n-1}\right) /\left(r_{n+1}-r_{n}\right)\right)=0
$$

since $\omega_{1}^{\prime}(\rho)<0$. Then, for $\varepsilon<1$, we would have

$$
r_{n+1}-r_{n}>(1 / \varepsilon)^{n-n_{0}}\left(r_{n_{0}}-r_{n_{0}-1}\right), \quad n \geqq n_{0} \text { for an } n_{0} .
$$

Letting $n \rightarrow \infty$, we obtain a contradiction. Hence we must have

$$
\lim _{n \rightarrow \infty} r_{n}=1 \text {. }
$$

Having proved that $r_{n} \uparrow 1$, we define

$$
\omega(r)=(1-r)\left(a_{n} r+b_{n}\right) \text { for } r_{n} \leqq r \leqq r_{n+1}, n=0,1, \cdots .
$$

Then, since

$$
a_{n+1}>a_{n} \text { and } \omega(r)>\sqrt{1-r}
$$

we have

$$
\omega(r) /(1-r) \text { is convex and } \omega(r) /(1-r) \uparrow \infty
$$

Further,

$$
\omega\left(r_{n}\right)=\omega_{1}\left(r_{n-1}\right), \quad \omega\left(r_{n+1}\right)=\omega_{1}\left(r_{n}\right) \downarrow 0
$$

and

$$
\omega(r)=v_{n}(r) \text { for } r_{n} \leqq r \leqq r_{n+1},
$$

where

$$
v_{n}(r)=-a_{n} r^{2}+\left(a_{n}-b_{n}\right) r+b_{n}
$$

Since

$$
v_{n}^{\prime}\left(r_{n}\right)=-2 a_{n} r_{n}+\left(a_{n}-b_{n}\right)=0,
$$

we get that

$\omega(r)$ is concave and monotone decreasing for $r_{n} \leqq r \leqq r_{n+1} \cdot$ Thus $\omega(r) \downarrow 0$, and our Lemma 1 is proved.

By the Lemma 1, functions $\omega(r)$ in the below may be supposed to satisfy the condition (3.1).

LEMmA 2. There exists a constant $K$ such that for any $z \in D$, $|z-\zeta| \leqq K \exp [-\omega(r) /(1-r)], r=|z|$, implies $\zeta \in D$ and moreover 


$$
\omega(\rho) /(1-\rho) \leqq 2 \omega(r) /(1-r), \quad \rho=|\zeta| \text {. }
$$

Proof. Since $\exp [-\omega(r) /(1-r)] \leqq \exp [-1 / \sqrt{1-r}]$, we have

$$
\exp [-\omega(r) /(1-r)] \leqq(1-r) / 2, \quad r \geqq R
$$

with an $R<1$. Put $K=(1-R) / 2$. Then we have that, if

$$
z \in D, \quad|z-\zeta| \leqq K \exp [-\omega(r) /(1-r)],
$$

we get

$$
\rho=|\zeta| \leqq(1+r) / 2<1 .
$$

If $\rho \geqq r$, we have, as $1-\rho \geqq(1-r) / 2$,

$$
\omega(\rho) /(1-\rho) \leqq 2 \omega(r) /(1-r) .
$$

If $\rho<r$, we have by (3.1)

$$
\omega(\rho) /(1-\rho) \leqq \omega(r) /(1-r) .
$$

$\left(3.6_{1-3}\right)$ give the lemma.

We note that $f \in F^{+}$implies $f^{\prime} \in F^{+}[9$, Theorem 6].

LEMMA 3. If $f$ is holomorphic in $D$, then $f$ belongs to $F^{+}$if and only if for some $\omega(r)$ satisfying (3.1)

$$
\left(\|f\|_{\omega}\right)^{2}=\frac{1}{\pi} \iint_{D}\left|f\left(r e^{i \theta}\right)\right|^{2} \exp \left[\frac{-\omega(r)}{1-r}\right] r d r d \theta<\infty .
$$

Proof. $f \in F^{+}$obviously satisfies (3.7) with some $\omega(r)$. On the other hand, it follows that the mean value of $|f|$ over the disk with center at $z \in D$ :

$$
\{\zeta ;|\zeta-z| \leqq K \exp [-\omega(r) /(1-r)]\} \subset D
$$

is bounded by

$$
(1 / K)\|f\|_{\omega} \exp [2 \omega(r) /(1-r)] \text {. }
$$

By the subharmonicity of $|f|$, this gives also a bound for $|f(z)|,|z|=r$, which shows that $f \in F^{+}$by (1.4).

LEMMA 4. Let $g$ be a form of type $(0,1)$ in $D$ with locally square summable coefficient $g(r, \theta)$, and let $\phi(r, \theta)$ be a subharmonic function in $D$ such that

$$
\iint_{D}|g(r, \theta)|^{2} e^{-\phi(r, \theta)} r d r d \theta<\infty .
$$

It follows that there is a function $f(a$ form of type $(0,0))$ with $\bar{\partial} f=g$, and 


$$
\begin{gathered}
\iint_{D}|f(r, \theta)|^{2} e^{-\phi(r, \theta)}\left(1+r^{2}\right)^{-2} r d r d \theta \\
\quad \leqq \iint_{D}|g(r, \theta)|^{2} e^{-\phi(r, \theta)} r d r d \theta
\end{gathered}
$$

Proof is found in [3, p. 945, Lemma 4].

For non-negative integers $p$ and $q$, we shall denote by $L_{q}^{p}$ the set of all differential forms $h$ of type $(0, q)$ with values in $\Lambda^{p} C^{N}$, such that for some function $\omega(r)$ satisfying (3.1),

$$
\iint_{D}|h(r, \theta)|^{2} \exp \left[\frac{-\omega(r)}{1-r}\right] r d r d \theta<\infty .
$$

In other words, for each $p$-tuple $S=\left(i_{1}, \cdots, i_{p}\right), 1 \leqq i_{1}, \cdots, i_{p} \leqq N, h$ has a component $h_{S}$ which is a differential form of type $(0, q)$ such that $h_{S}$ is skew symmetric in $S$ and

$$
\iint_{D}\left|h_{S}(r, \theta)\right|^{2} \exp \left[\frac{-\omega(r)}{1-r}\right] r d r d \theta<\infty \text {. }
$$

Note that $L_{q}^{p}=0$ if $p>N$ or $q>1$.

Now $\bar{\partial}$-operator acts componentwise on the elements of $L_{q}^{p}$ and yields a linear mapping $\bar{\partial}: L_{q}^{p} \rightarrow\left\{(0, q+1)\right.$-forms with values in $\left.\Lambda^{p} C^{N}\right\}$, such that $\bar{\partial}^{2}=0$. Furthermore, the interior product $P_{f}$ by $\vec{f}=\left(f_{1} \cdots, f_{N}\right)$ maps $L_{q}^{p+1}$ into $L_{q}^{p}$ : If $h \in L_{q}^{p+1}$ then

$$
\left(P_{f} h\right)_{S}=\sum_{j=1}^{N} h_{S j} f_{j} \quad \text { for } \quad S=\left(i_{1}, \cdots, i_{p}\right) .
$$

We define $P_{f} L_{q}^{0}=0$. Clearly $P_{f}^{2}=0$ and $P_{f}$ commutes with $\bar{\partial}$ since $f_{1}, \cdots, f_{N}$ are holomorphic. So, we have a double complex.

LEMMA 5. For every $h \in L_{1}^{p}$, the equation $\bar{\partial} g=h$ has a solution $g \in L_{0}^{p}$.

Proof. This follows immediately from the Lemma 4.

Lemma 6. For any $v \in C^{2}(D)$ we have for $0 \leqq r<1$,

$$
\int_{0}^{r} t^{-1} S(t) d t=\frac{1}{2 \pi} \int_{0}^{2 \pi} v(r, \theta) d \theta-v(0),
$$

where

$$
S(t)=\frac{1}{2 \pi} \iint_{|z| \leqq t} \Delta v d x d y, \quad \Delta v=\frac{\partial^{2} v}{\partial x^{2}}+\frac{\partial^{2} v}{\partial y^{2}} .
$$

Proof is a simple consequence of Green's formula. See [4, p. 231, Lemma 3.3]. 
Lemma 7. Let $f_{1}, \cdots, f_{N} \in F^{+}$. Then, if we put

$$
w_{i j}(z)=\left(f_{i}(z) f_{j}^{\prime}(z)-f_{j}(z) f_{i}^{\prime}(z)\right) /\|\vec{f}(z)\|^{2}, \quad i, j=1, \cdots, N,
$$

we have

$$
\iint_{D}\left|w_{i j}(z)\right|^{2} \exp \left[\frac{-\omega(r)}{1-r}\right] r d r d \theta<\infty
$$

for a function $\omega(r)$ satisfying (3.1).

Proof. At first we suppose that $f_{1}, \cdots, f_{N}$ have no common zeros. Then

$$
v(z)=2 \log \|\vec{f}(z)\| \in C^{2}(D),
$$

and, if we write

$$
w(z)=\frac{1}{4} \Delta v=\sum_{i, j=1}^{N}\left|f_{i}(z) f_{j}^{\prime}(z)-f_{j}(z) f_{i}^{\prime}(z)\right|^{2} /\|\vec{f}(z)\|^{4},
$$

it suffices to prove that

$$
\iint_{D} w(z) \exp \left[\frac{-\omega(r)}{1-r}\right] r d r d \theta<\infty .
$$

We apply Lemma 6 for the function $v$ in (3.10). Then

$$
\int_{0}^{r} t^{-1} S(t) d t \leqq \frac{\lambda(r)}{1-r}
$$

for a continuous function $\lambda(r)$ satisfying (3.1). Now $S(t)$ is non-negative and increasing, since $v(z)$ in (3.10) is subharmonic, so

$$
\int_{0}^{r} \frac{S(t)}{t} d t \geqq \int_{r^{2}}^{r} \frac{S(t)}{t} d t \geqq \frac{S\left(r^{2}\right)}{r} r(1-r),
$$

thus

$$
S\left(r^{2}\right) \leqq \frac{\lambda(r)}{(1-r)^{2}} \leqq \frac{4 \lambda(r)}{\left(1-r^{2}\right)^{2}} \text { hence } S(r) \leqq\left(\frac{\omega(r)}{1-r}\right)^{2}
$$

with a continuous function $\omega(r)$ satisfying (3.1).

Then, writing $\omega(r) /(1-r)=p(r)$,

$$
\begin{aligned}
& \iint_{D} w(z) \exp \left[\frac{-\omega(r)}{1-r}\right] r d r d \theta \leqq \iint_{p(r) \leqq 2}+\sum_{n=1}^{\infty} \iint_{2^{n} \leqq p(r) \leqq 2^{n+1}} \\
& \quad \leqq K_{0}+\sum_{n=1}^{\infty} e^{-2^{n}} \iint_{p(r) \leqq 2^{n+1}} \Delta v d x d y \\
& \quad \leqq K_{0}+\sum_{n=1}^{\infty} 2^{2 n+2} \exp \left[-2^{n}\right]<\infty .
\end{aligned}
$$


For the case where $f_{1}, \cdots, f_{N}$ have common zeros, the desired conclusion may be deduced via a standard argument by considering $v_{c}(z)=$ $\log \left(\|\vec{f}(z)\|^{2}+\varepsilon^{2}\right)$ and letting $\varepsilon \rightarrow 0$.

q.e.d.

Proof of Theorem 1. Suppose $u \in F^{+}$satisfies (2.2). Let $\alpha=$ $\left(\alpha_{1}, \cdots, \alpha_{N}\right) \in L_{0}^{1}$ be such that

$$
\alpha_{i}=u^{2} \bar{f}_{i} /\|\vec{f}\|^{2}, \quad i=1, \cdots, N .
$$

Then

$$
\bar{\partial} \alpha_{i}=\|\vec{f}\|^{-4} u^{2} \sum_{j=1}^{N} f_{j} \overline{\left(f_{j} \partial f_{i}-f_{i} \partial f_{j}\right)} .
$$

If we put

$$
\beta_{i j}=\|\vec{f}\|^{-4} u^{2} \overline{\left(f_{j} \partial f_{i}-f_{i} \partial f_{j}\right)},
$$

then we get $\beta=\left(\beta_{i j}\right) \in L_{1}^{2}$ by Lemma 7. Clearly, $\bar{\partial} \alpha=P_{f} \beta$, and there exists $\gamma \in L_{0}^{2}$ with $\bar{\partial} \gamma=\beta$ by Lemma 5 . Then, if we put

$$
g=\alpha-P_{f} \gamma \in L_{0}^{1},
$$

then $\bar{\partial} g=0$, hence $g_{j} \in F^{+}, j=1, \cdots, N$, by Lemma 3 , and

$$
P_{f} g=u^{2} \text {, which shows that } u^{2} \in I\left(f_{1}, \cdots, f_{N}\right) \text {. q.e.d. }
$$

\section{Proofs of Theorem $1^{*}, 2^{*}$ and 3 .}

Proof of Theorem $1^{*}$. Let $f, g \in F^{+}$. If we take in (2.2) $N=2$, $f_{1}=f^{2}, f_{2}=g^{2}$ and $u=f g$, then (2.2) holds. If it were true that (2.2) would imply $u \in I\left(f_{1}, \cdots, f_{N}\right)$, we would have $f g \in I\left(f^{2}, g^{2}\right)$ for any $f, g \in F^{+}$. We will show that this is not the case for some $f$ and $g$.

Suppose $f g \in I\left(f^{2}, g^{2}\right)$, i.e., $f g=A f^{2}+B g^{2}$ with $A, B \in F^{+}$. Then

$$
A f^{2} / g=f-B g \in F^{+}, \quad B g^{2} / f=g-A f \in F^{+} .
$$

We put

$$
f(z)=\prod_{k=1}^{\infty}\left(\left(z-z_{k}\right) /\left(1-\bar{z}_{k} z\right)\right),
$$

where

$$
z_{k}=1-b^{k} \quad \text { with a constant } b, 0<b<1 / 3,
$$

and

$$
g(z)=\exp \left[-c \frac{1+z}{1-z}\right] \text { with a constant } c>0 .
$$

Then by (4.1), $B / f$ is holomorphic.

Then, as we shall see shortly later, if $H(z)$ is holomorphic in $D$,

$$
f \times H \in F^{+} \text {implies } H \in F^{+} \text {. }
$$


Thus

$$
\begin{array}{lll}
A / g=p \in F^{+} & \text {since } & (A / g) f^{2} \in F^{+}, \\
B / f=q \in F^{+} & \text {since } & B \in F^{+} .
\end{array}
$$

Hence

$$
1=p \times f+q \times g .
$$

This is impossible, since $p\left(z_{k}\right) f\left(z_{k}\right)=0$ and $q\left(z_{k}\right) g\left(z_{k}\right) \rightarrow 0$ as seen from (1.4) and the definition of $g(z)$.

Now we will show (4.2). First we note that

$$
|f(z)| \geqq \Pi|| z|-| z_{k}|| /\left(1-\left|z_{k}\right||z|\right) .
$$

Put

$$
r^{(n)}=1-b^{n}(1+b) / 2=\left(z_{n}+z_{n+1}\right) / 2 .
$$

Then, if $|z|=r^{(n)}$,

$$
\begin{gathered}
|| z|-| z_{k}||=\left|b^{k}-b^{n}(1+b) / 2\right|, \\
1-\left|z_{k}\right||z| \leqq b^{k}+b^{n}(1+b) / 2 .
\end{gathered}
$$

Thus, if $|z|=r^{(n)}$,

$$
\begin{aligned}
|f(z)| & =\prod_{k \leqq n} \prod_{k>n} \geqq \prod_{k \geqq n} \frac{1-b^{n-k}(1+b) / 2}{1+b^{n-k}(1+b) / 2} \prod_{k>n} \frac{1-b^{k-n} \times 2 /(1+b)}{1+b^{k-n} \times 2 /(1+b)} \\
& \geqq \prod_{m \geqq 0} \frac{1-b^{m}(1+b) / 2}{1+b^{m}(1+b) / 2} \prod_{m \geq 0} \frac{1-b^{m} \times 2 b /(1+b)}{1+b^{m} \times 2 b /(1+b)}=K>0 .
\end{aligned}
$$

Let

$$
h(z)=f(z) H(z) \in F^{+} .
$$

Then, for any constant $a>0$,

$$
M(r, h) \exp \left[\frac{-a}{1-r}\right] \rightarrow 0 \quad \text { as } r \rightarrow 1
$$

By (4.4), we have

$$
M\left(r^{(n)}, H\right) \leqq M\left(r^{(n)}, h\right) / K .
$$

Thus, for $r^{(n-1)} \leqq r \leqq r^{(n)}$

$$
M(r, H) \leqq M\left(r^{(n)}, h\right) / K .
$$

Hence, for $r^{(n-1)} \leqq r \leqq r^{(n)}$,

$$
M(r, H) \exp \left[\frac{-a}{1-r}\right] \leqq K^{-1} M\left(r^{(n)}, h\right) \exp \left[-a /\left(1-r^{(n-1)}\right)\right]
$$




$$
\leqq K^{-1} M\left(r^{(n)}, h\right) \exp \left[-a b /\left(1-r^{(n)}\right)\right] \rightarrow 0 \quad \text { as } n \rightarrow \infty \text {, }
$$

hence

$$
M(r, H) \exp \left[\frac{-a}{1-r}\right] \rightarrow 0 \quad \text { as } \quad r \rightarrow 1
$$

for any $a>0$, which shows that $H \in F^{+}$.

q.e.d.

Proof of Theorem $2 *$. Let $\nu(t), 0 \leqq t \leqq 2 \pi$, be a continuous and monotone increasing function such that $\nu(0)=0, \nu(2 \pi)=1$, and $\nu^{\prime}(t)=0$ almost everywhere on $[0,2 \pi]$. We put, for $2 n \pi \leqq t \leqq(2 n+2) \pi$,

$$
\mu(t)=n+\nu(t-2 n \pi), \quad n=0, \pm 1, \pm 2, \cdots
$$

and

$$
f(z)=\exp \left[-\int_{0}^{2 \pi} \frac{e^{i t}+z}{e^{i t}-z} d \mu(t)\right] .
$$

Then $f(z) \in H^{\infty} \subset N^{+}$and

$$
1 / f(z) \in F^{+},
$$

as shown in [12, Proof of Theorem 1]. Therefore, $f(z)$ satisfies (2.4) but does not generate $N^{+}$, since $f(z)$ is not invertible in $N^{+}$while $f(z)$ is invertible in $F^{+}$.

Proof of Theorem 3. Put

$$
E=\left\{\exp \left[-c \frac{1+z}{1-z}\right] ; c>0\right\}
$$

Then $E \subset N^{+} \subset F^{+}$. If we write $I=\bigcup_{f \in F^{+}} f E, I$ is a proper ideal. It is easy to see that

$$
\exp \left[-c \frac{1+z}{1-z}\right] \rightarrow 1 \text { as } c \rightarrow 0
$$

Hence the maximal ideal containing $I$ is not closed.

5. Closed maximal ideals in $\boldsymbol{F}^{+}$. Let $A$ be a topological algebra with identity 1 , locally convex and commutative, over the complex number field $C$. Topology of $A$ is defined by a countable family of semi-norms $\left\{\|\cdot\|_{\alpha \in I}\right.$, which are supposed to satisfy that $\|1\|_{\alpha}=1$ and for $a, b \in A$

$$
\|a b\|_{\alpha} \leqq\|a\|_{\alpha}\|b\|_{\alpha} \text { for every } \alpha \in I \text {. }
$$

For an $\alpha \in I$, let $E_{\alpha}=\left\{a \in A ;\|a\|_{\alpha}=0\right\} . \quad E_{\alpha}$ is obviously an ideal in $A$. For $a \in A$, we write $a^{\wedge}=a+E_{\alpha} \in A / E_{\alpha}$. Then $A / E_{\alpha}$ is a normed space with $\left\|a^{\wedge}\right\|_{\alpha}=\|a\|_{\alpha}$. We have, by (5.1), for $a, b \in A$ 


$$
\left\|a^{\wedge} b^{\wedge}\right\|_{\alpha} \leqq\left\|a^{\wedge}\right\|_{\alpha}\left\|b^{\wedge}\right\|_{\alpha} .
$$

The completion of $A / E_{\alpha}$ with res. to the norm $\|\cdot\|_{\alpha}$ is denoted as $A_{\alpha}^{*}$.

Lemma 8. Let $g \in A$. Suppose $\mid 1-\mu g$ is invertible for $|\mu|<\delta$. Then, for any $\alpha \in I$ there is a $\delta(\alpha)>0$ such that $\left\|(1-\mu g)^{-1}\right\|_{\alpha}$ is bounded for $|\mu| \leqq \delta(\alpha)$.

Proof. Put $\delta(\alpha)=\min \left(\delta, 1 / 2\|g\|_{\alpha}\right)$ and

$$
h_{n}=1+\mu g+\cdots+\mu^{n} g^{n} \in A, \quad h_{n}^{\wedge} \in A / E_{\alpha} .
$$

$\left\{h_{n}^{\wedge}\right\}$ is a Cauchy sequence in $A_{\alpha}^{*}$ if $|\mu| \leqq \delta(\alpha)$. Then

$$
h^{\wedge}=\lim _{n \rightarrow \infty} h_{n}^{\wedge} \in A_{\alpha}^{*} \text {. }
$$

For a fixed $\mu$, we define a linear operator $T$ on $A / E_{\alpha}$ by

$$
T a^{\wedge}=(1-\mu g)^{\wedge} a^{\wedge} \in A / E_{\alpha} \text { for } a \in A .
$$

$T$ is continuous on $A / E_{\alpha}$ by $\left(5.1^{\prime}\right)$, and continuously extended on $A_{\alpha}^{*}$. Then

$$
T h^{\wedge}=\lim _{n \rightarrow \infty} T h_{n}^{\wedge}=\lim _{n \rightarrow \infty}\left((1-\mu g) h_{n}\right)^{\wedge}=\lim _{n \rightarrow \infty}\left(1-\mu^{n+1} g^{n+1}\right)^{\wedge}=1^{\wedge} .
$$

Thus $h^{\wedge}=\left((1-\mu g)^{-1}\right)^{\wedge} \in A / E_{\alpha}$. Then we have, for $|\mu| \leqq \delta(\alpha)$

$$
\left\|(1-\mu g)^{-1}\right\|_{\alpha}=\lim _{n \rightarrow \infty}\left\|h_{n}^{\hat{n}}\right\|_{\alpha}=\lim _{n \rightarrow \infty}\left\|h_{n}\right\|_{\alpha} \leqq 1+\sum_{n=1}^{\infty}\|\mu g\|_{\alpha}^{n} \leqq 2 .
$$

Lemma 9. Let $f \in A$ and $\lambda_{0} \in C$. Suppose $\lambda-f$ is invertible for $\left|\lambda-\lambda_{0}\right|<\delta, \delta>0$. Then $(\lambda-f)^{-1}$ is continuous with respect to $\lambda$.

Proof. Put $\left(\lambda_{0}-f\right)^{-1}=g$ and $\mu=\lambda_{0}-\lambda$. Then

$$
\begin{aligned}
& (\lambda-f)^{-1}-\left(\lambda_{0}-f\right)^{-1} \\
= & \left(\lambda_{0}-f\right)^{-1}\left[(1-\mu g)^{-1}-1\right] \\
= & \mu g\left(\lambda_{0}-f\right)^{-1}(1-\mu g)^{-1}=\mu g^{2}(1-\mu g)^{-1} .
\end{aligned}
$$

Then for any $\alpha \in I$, if $|\mu| \leqq \delta(\alpha)$,

$$
\begin{aligned}
& \left\|(\lambda-f)^{-1}-\left(\lambda_{0}-f\right)^{-1}\right\|_{\alpha} \\
\leqq & |\mu|\|g\|_{\alpha}^{2}\left\|(1-\mu g)^{-1}\right\|_{\alpha} \\
\leqq & 2|\mu|\|g\|_{\alpha}^{2} \rightarrow 0 \text { as } \lambda \rightarrow \lambda_{0}, \quad \mu \rightarrow 0,
\end{aligned}
$$

hence $(\lambda-f)^{-1}$ is continuous.

Lemma 10. For any $f \in A$, there is a number $\lambda_{f}$ such that $\lambda_{f}-f$ is not invertible.

PRoof. Suppose $\lambda-f$ were invertible for any $\lambda \in C$. Then $(\lambda-f)^{-1}$ 
is continuous with respect to $\lambda$. Let $L$ be a continuous linear functional on $A$. Then

$$
G(\lambda)=L\left((\lambda-f)^{-1}\right)
$$

is an entire function. For,

$$
G(\lambda)-G\left(\lambda_{0}\right)=-\left(\lambda-\lambda_{0}\right) L\left((\lambda-f)^{-1}\left(\lambda_{0}-f\right)^{-1}\right),
$$

hence, by Lemma 9 , we obtain

$$
G^{\prime}\left(\lambda_{0}\right)=-L\left(\left(\lambda_{0}-f\right)^{-1}\left(\lambda_{0}-f\right)^{-1}\right) .
$$

Further, by the continuity of $L$,

$$
|G(\lambda)|=\left|L\left((\lambda-f)^{-1}\right)\right| \leqq K\left\|(\lambda-f)^{-1}\right\|_{\alpha}
$$

with an $\alpha \in I$ and a constant $K$. Thus, by Lemma 8 , if $|\lambda|>2\|f\|_{\alpha}$,

$$
|G(\lambda)| \leqq K|\lambda|^{-1}||(1-f / \lambda)^{-1} \|_{\alpha} \leqq 2 K /|\lambda| \rightarrow 0
$$

as $|\lambda| \rightarrow \infty$. Therefore $G(\lambda) \equiv 0$, i.e.,

$$
L\left((\lambda-f)^{-1}\right) \equiv 0 \quad \text { for } \quad \lambda \in C
$$

for any continuous linear functional $L$ on $A$, which is absurd.

As a characterization of closed maximal ideals we have, in analogy with the well known theorem of Igusa [4], the following

THeORem 4. Let $M$ be a maximal ideal in $F^{+}$. The following conditions for $M$ are equivalent:

(i) $M$ is closed in the topology of uniform convergence on every disk $|z| \leqq r, r<1$.

(ii) $F^{+} / M \cong C$.

(iii) $M$ corresponds to a point $z_{0} \in D$, i.e., $M$ consists of all functions of $F^{+}$which vanish at $z_{0}$.

Proof. (i) $\rightarrow$ (ii): Obviously, $F^{+} / M \supset C$. For $f \in F^{+}$, we denote

$$
[f]=f+M \in F^{+} / M \text {. }
$$

We introduce the family of semi-norms in $F^{+} / M$ as follows:

$$
\|[f]\|_{r}=\inf _{h \in M}\left(\max _{|z|=r}|f(z)+h(z)|\right), \quad 0 \leqq r<1 .
$$

Then clearly

$$
\|[f g]\|_{r} \leqq\|[f]\|_{r}\|[g]\|_{r}, \quad 0 \leqq r<1 .
$$

By Lemma 10, to each $[f] \in F^{+} / M$, there corresponds a number $\lambda \in C$ such that $\lambda-[f]$ is not invertible. But, since $F^{+} / M$ is a field by the maximality of $M, \lambda-f$ must belong to $M$, i.e., $\lambda \in[f]$. Thus we obtain 


$$
F^{+} / M \cong C
$$
$z_{0} \in D$.

(ii) $\rightarrow$ (iii): Let $z_{0}$ be the coset $[z] \in F^{+} / M$. Then $z-z_{0} \in M$, hence

For each $f(z) \in F^{+}$, we have

$$
f(z)-f\left(z_{0}\right)=A(z)\left(z-z_{0}\right) .
$$

As easily seen, $A(z) \in F^{+}$, thus $f(z)-f\left(z_{0}\right) \in M$. If $f(z) \in M$, then $f\left(z_{0}\right) \in M$, whence $f\left(z_{0}\right)=0$. Thus $M$ corresponds to the point $z_{0} \in D$.

(iii) $\rightarrow$ (i): This is evident from the theorem of Hurwitz.

6. Maximal ideals in $\boldsymbol{F}^{+}$. Now we will study some structures of maximal ideal space of the algebra $F^{+}$.

The complex $w$-sphere is denoted by $W$. Let $Q$ be the set of all continuous functions $\omega(r), 0 \leqq r<1$, satisfying (3.1).

Taking a function $f(z) \in F^{+}$, we define a topology $\tau_{Q}(f)$ in $W$.

For a number $\varepsilon>0$ and a function $\omega(r) \in Q$, we define neighborhood $U(a)$ of $a \in W$ as follows:

(A) $a \neq \infty$.

A(i) Suppose there is a point $z_{0} \in D$ such that $f\left(z_{0}\right)=a$. Then we put for a number $\eta>0$,

$$
\begin{aligned}
U(a)= & U\left(a ; \varepsilon, \omega, z_{0}, \eta\right)=\left\{w ; w=f(z), \text { where }\left|z-z_{0}\right|<\eta\right. \text { and } \\
& \left.\exp \left[\frac{\omega(|z|)}{1-|z|}\right]|f(z)-a|<\varepsilon\right\}
\end{aligned}
$$

A(ii) Suppose there is a point $\zeta_{0},\left|\zeta_{0}\right|=1$, such that

$$
\varliminf_{z \rightarrow \zeta_{0}} \exp \left[\frac{\omega(|z|)}{1-|z|}\right]|f(z)-a|=0 \text {. }
$$

Then we put for a number $\eta>0$,

$$
\begin{aligned}
U(a)= & U\left(a ; \varepsilon, \omega, \zeta_{0}, \eta\right)=\left\{w ; w=a+\rho e^{i \theta}, 0 \leqq \theta \leqq 2 \pi,\right. \text { and } \\
& \rho<\varepsilon \exp \left[\frac{-\omega(|z|)}{1-|z|}\right] \text { for a point } z \in D \text { such that } \\
& \left.\left|z-\zeta_{0}\right|<\eta, \exp \left[\frac{\omega(|z|)}{1-|z|}\right]|f(z)-a|<\varepsilon\right\} \cup\{a\} .
\end{aligned}
$$

A(iii) Suppose there is neither $z_{0}$ in $\mathrm{A}(\mathrm{i})$ nor $\zeta_{0}$ in $\mathrm{A}(\mathrm{ii})$. We put

$$
U(a)=U(a ; \varepsilon, \omega)=\{a\} \text {. }
$$

(B) $\quad a=\infty$. 
B(i) Suppose there is a point $\zeta_{0},\left|\zeta_{0}\right|=1$, such that

$$
\varlimsup_{z \rightarrow \zeta_{0}} \exp \left[\frac{-\omega(|z|)}{1-|z|}\right]|f(z)|=\infty .
$$

Then we put for a number $\eta>0$,

$$
\begin{aligned}
U(\infty)= & U\left(\infty ; \varepsilon, \omega, \zeta_{0}, \eta\right)=\left\{w ; w=\rho e^{i \theta}, 0 \leqq \theta \leqq 2 \pi,\right. \text { and } \\
& \rho>(1 / \varepsilon) \exp \left[\frac{\omega(|z|)}{1-|z|}\right] \text { for a point } z \in D \text { such that } \\
& \left.\left|z-\zeta_{0}\right|<\eta, \exp \left[\frac{-\omega(|z|)}{1-|z|}\right]|f(z)|>1 / \varepsilon\right\} \cup\{\infty\} .
\end{aligned}
$$

$\mathrm{B}$ (ii) Suppose there is no point $\zeta_{0}$ in $\mathrm{B}$ (ii). Then

$$
U(\infty)=U(\infty ; \varepsilon, \omega)=\{\infty\} .
$$

By this system of neighborhoods, $W$ becomes a Hausdorff space. We note that the topology depends on the function $f(z)$.

Let $f(D) \subset W$ be the range of $f(z)$ in $D$, and $(f(D))^{a}$ be the closure of $f(D)$ with respect to the topology determined by $f$. Since $f(z) \in F^{+}$, $(f(D))^{a}$ does not contain $\infty$. We compactify $(f(D))^{a}$ as follows:

Let $P_{f}$ be an (abstract) element. Neighborhoods of $P_{f}$ are defined to be open sets (in the sense of the usual Riemann sphere topology) containing $W-(f(D))^{a}$.

Then, $A_{f}=(f(D))^{a} \cup\left\{P_{f}\right\}$ is obviously compact. We note that $A_{f}-\left\{P_{f}\right\}$ satisfies the Hausdorff separation axiom, although $A_{f}$ does not. $A_{f}$ might be considered, in a sense, as an Alexandroff compactification of $(f(D))^{a}$.

Further, let $C_{0}$ be the set of all continuous complex valued functions with compact supports in $D$.

Put

$$
T=\prod_{f \in F^{+}} A_{f} \cdot \prod_{\phi \in C_{0}} W_{\phi}\left(W_{\phi}=\underset{\text { sphere topology })}{W \text { with the usual Riemann }}\right.
$$

$T$ is compact with the Tychonoff topology. We denote by $\pi_{f}$ or $\pi_{\phi}$ the projection of $T$ on $A_{f}$ or on $W_{\phi}$, respectively. We write, for $z \in D$,

$$
\psi(z)=\{f(z), \phi(z)\}_{f \in F^{+}, \phi \in C_{0}}
$$

$\psi$ is a continuous and one-to-one mapping from $D$ into $T$. We write the closure of $\psi(D)$ in $T$ as $D^{*}$. Then $D^{*}$ is compact and $\psi(D)$ is dense in $D^{*}$. $\psi$ is an open mapping. To see this, for $z_{0} \in D$, let $U$ be a relatively compact neighborhood of $z_{0}$, and $\phi$ be a function of $C_{0}$ with support 
contained in $U$ and $\phi\left(z_{0}\right) \neq 0$. Put

$$
V=\left\{p \in D^{*} ; \pi_{\phi}(p) \neq 0\right\} \text {. }
$$

$V$ is a neighborhood of $\psi\left(z_{0}\right)$ on $D^{*} . \quad V-\psi(\bar{U})$ is an open set, as we shall see shortly later. But we have

$$
(V-\psi(\bar{U})) \cap \psi(D)=\text { void }
$$

hence $V-\psi(\bar{U})$ is void, for $\psi(D)$ is dense in $D^{*}$. Therefore we obtain

$$
V \subset \psi(\bar{U}) \subset \psi(D), \quad V \subset \psi(U),
$$

and $\psi$ is an open mapping.

Now we will show that $V-\psi(\bar{U})$ is open, i.e., $\psi(\bar{U})$ is closed. Let $q \notin \psi(\bar{U})$. If there is an $f_{0} \in F^{+}$such that $\pi_{f_{0}}(q) \neq P_{f_{0}}$, then there is a neighborhood $U\left(\pi_{f_{0}}(q)\right)$ such that $U\left(\pi_{f_{0}}(q)\right) \cap \pi_{f_{0}}(\psi(\bar{U}))=$ void. If $\pi_{f}(q)=P_{f}$ for any $f \in F^{+}$, then there is, for an $f_{0} \in F^{+}$, a neighborhood $U\left(P_{f_{0}}\right)$ such that $U\left(P_{f_{0}}\right) \cap \pi_{f_{0}}(\psi(\bar{U}))=$ void, since $\pi_{f_{0}}(\psi(\bar{U}))=f_{0}(\bar{U})$ is compact in $f_{0}(D)$. Thus, if $U(q)$ is a neighborhood of $q$ such that $\pi_{f_{0}}(U(q))=U\left(\pi_{f_{0}}(q)\right)$, then $U(q) \cap \psi(\bar{U})=$ void, and $(\psi(\bar{U}))^{C}$ is open, hence $\psi(\bar{U})$ is closed.

Thus $\psi$ is homeomorphic, and $D$ and $\psi(D)$ may be identified.

$\pi_{f}$ is the continuous extension of $f$ onto $D^{*}$. For $a, b \in D^{*}, a \neq b$, there is an $f \in F^{+}$with

$$
\pi_{f}(a) \neq \pi_{f}(b),
$$

since for any point $p \in D^{*}-\psi(D)$ we have $\phi(p)=0$ for each $\phi \in C_{0}$.

We put

$$
P=\prod_{f \in F^{+}}\left\{P_{f}\right\} \cdot \prod_{\phi \in C_{0}} W_{\phi}
$$

and

$$
D^{* *}=D^{*}-P \text {. }
$$

Let $\mathfrak{M}$ be the set of all maximal ideals in $F^{+}$. Then

THEOREM 5. Elements of $\mathfrak{M}$ and points of the space $D^{* *}$ correspond in a one-to-one way.

Proof. Let $z_{0}$ be a point of $D$. It is easy to see that the set of all functions $f(z) \in F^{+}$with $f\left(z_{0}\right)=0$ forms a maximal ideal in $F^{+}$.

Let $J$ be a maximal ideal in $F^{+}$. We suppose that there are no common zeros in $D$ for functions of $J$.

Let $f_{\alpha_{1}}, \cdots, f_{\alpha_{N}}$ be functions of $J$. Thus, by Theorem 2, we have for any $\omega(r) \in Q$,

$$
\inf _{r_{0} \leqq r<1} \exp [\omega(r) /(1-r)]\left(\left|f_{\alpha_{1}}(z)\right|+\cdots+\left|f_{\alpha_{N}}(z)\right|\right)=0
$$


$(r=|z|)$ for any $r_{0}<1$. Thus, there is a sequence $\left\{z_{n}\right\} \subset D, r_{n}=\left|z_{n}\right| \rightarrow 1$, such that

$$
\liminf _{n \rightarrow \infty} \exp \left[\omega\left(r_{k}\right) /\left(1-r_{k}\right)\right]\left(\left|f_{\alpha_{1}}\left(z_{k}\right)\right|+\cdots+\left|f_{\alpha_{N}}\left(z_{k}\right)\right|\right)=0 .
$$

We denote by $E\left(\alpha_{1}, \cdots, \alpha_{N} ; \omega\right)$ the set of points of $D^{*}$ such that

$$
\begin{aligned}
& \zeta^{*} \in E\left(\alpha_{1}, \cdots, \alpha_{N} ; \omega\right) \text { if for any neighborhood } U\left(\zeta^{*}\right), \\
& \inf _{z \in U\left(\zeta^{*} \cap \cap D\right.} \exp [\omega(|z|) /(1-|z|)]\left(\left|f_{\alpha_{1}}(z)\right|+\cdots+\left|f_{\alpha_{N}}(z)\right|\right)=0 .
\end{aligned}
$$

$E\left(\alpha_{1}, \cdots, \alpha_{N} ; \omega\right)$ is a closed non-void subset of the compact space $D^{*}$, for every $\omega(r) \in Q$, by Theorem 2 , since $J$ is a proper ideal. If $\omega_{1}(r), \cdots$, $\omega_{M}(r) \in Q$, we have

$$
\begin{gathered}
E\left(\alpha_{1}, \cdots, \alpha_{N} ; \omega_{1}\right) \cap \cdots \cap E\left(\alpha_{1}, \cdots, \alpha_{N} ; \omega_{M}\right) \\
\quad \supset E\left(\alpha_{1}, \cdots, \alpha_{N} ; \omega_{1}+\cdots+\omega_{M}\right) \neq \text { void } .
\end{gathered}
$$

Hence

$$
E\left(\alpha_{1}, \cdots, \alpha_{N}\right)=\bigcap_{\omega \in Q} E\left(\alpha_{1}, \cdots, \alpha_{N} ; \omega\right)
$$

is non-void. Since

$$
E\left(\alpha_{1}, \cdots, \alpha_{N}\right) \cap E\left(\alpha_{1}^{\prime}, \cdots, \alpha_{K}^{\prime}\right) \supset E\left(\alpha_{1}, \cdots, \alpha_{N}, \alpha_{1}^{\prime}, \cdots, \alpha_{K}^{\prime}\right) \neq \text { void },
$$

we have

is non-void.

$$
E=\bigcap_{\left(\alpha_{1}, \cdots, \alpha_{N}\right)} E\left(\alpha_{1}, \cdots, \alpha_{N}\right)
$$

Let $\zeta^{*} \in E$ and $M\left(\zeta^{*}\right)$ be the set of all functions $f \in F^{+}$such that

$$
\exp \left[\frac{\omega(|z|)}{1-|z|}\right]|f(z)| \rightarrow 0 \text { for each } \omega(r) \in Q,
$$

as $z \rightarrow \zeta^{*}$ in $D^{*}, z \in D$.

$M\left(\zeta^{*}\right)$ is obviously a proper ideal. Take a function $f \in J$. For any $\varepsilon>0$ and $\omega(r) \in Q$, we choose a neighborhood $U\left(\zeta^{*}\right)$ as

$$
\pi_{f}\left(U\left(\zeta^{*}\right)\right)=U\left(\pi_{f}\left(\zeta^{*}\right) ; \varepsilon, \omega, \zeta_{0}, \eta\right)
$$

as defined in A(ii) with suitable $\zeta_{0},\left|\zeta_{0}\right|=1$, and $\eta>0$. Thus, if $z \in U\left(\zeta^{*}\right)$,

$$
\exp [\omega(|z|) /(1-|z|)]\left|f(z)-\pi_{f}\left(\zeta^{*}\right)\right|<\varepsilon .
$$

But, by the definition of the set $E$, we have $\pi_{f}\left(\zeta^{*}\right)=0$, hence $f$ satisfies (6.4) and $J \subset M\left(\zeta^{*}\right)$, hence $J=M\left(\zeta^{*}\right)$.

We have that $E \subset D^{* *}$. $E$ contains only one point, since the extensions of functions of $F^{+}$separate points of $D^{*}$. Thus we obtain the proof of our theorem. 


\section{REFERENCES}

[1] N. Dunford and J. T. Schwartz, Linear operators, part I, Interscience Publishers Inc., New York, 1964.

[2] P. L. DuREN, Theory of $H^{p}$ spaces, Academic Press, New York and London, 1970.

[3] L. Hörmander, Generators for some rings of analytic functions, Bull. Amer. Math. Soc., 73 (1967), 943-949.

[4] J. IGUSA, On a property of the domain of regularity, Mem. Coll. Sci. University of Kyoto, Ser. A, 27 (1952), 95-97.

[5] J. J. KeLLEHeR AND B. A. TAYLOR, Finitely generated ideals in rings of analytic functions, Math. Ann., 193 (1971), 225-237.

[6] J. J. KeLleHER AND B. A. TAYLOR, Closed ideals in locally convex algebras of analytic functions, J. Reine Angew. Math., 255 (1972), 190-209.

[7] G. Ко̆тну, Topologische Lineare Räume I, Zweite Auflage, Springer-Verlag, BerlinHeidelberg-New York, 1966.

[8] K. V. RaJeswara Ra0, On a generalized corona problem, J. Analyse Math., 18 (1967), 277-278.

[9] N. Yanaginara, Multipliers and linear functionals for the class $N^{+}$, Trans. Amer. Math. Soc., 180 (1973), 449-461.

[10] N. Yanaginara, The containing Fréchet space for the class $N^{+}$, Duke Math. J., 40 (1973), 93-103.

[11] N. Yanaginara, The second dual space for the space $N^{+}$, Proc. Japan Acad., 49 (1973), 33-36.

[12] N. Yanagihara, The class $N^{+}$of holomorphic functions and its containing Fréchet space $F^{+}$, Boll. Un. Mat. Ital. (Bologna), 8 (1973), 230-245.

Department of Mathematics, Chiba University,

1-33 Yayoi-cho, Chiba City, Chiba-ken, JaPan. 
